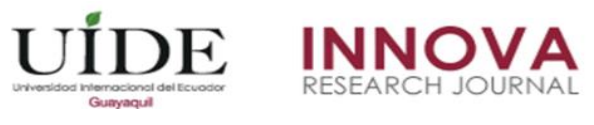

INNOVA Research Journal, ISSN 2477-9024

(Julio, 2017). Vol. 2, No.7 pp. 121-132

DOI: https://doi.org/10.33890/innova.v2.n7.2017.238

URL: http://revistas.uide.edu.ec/index.php/innova/index

Correo: innova@uide.edu.ec

\title{
Vinculación entre las políticas públicas de CT+I y de propiedad intelectual: herramientas para el desarrollo
}

\section{Linkage between the public policies of $\mathrm{CT}+\mathrm{I}$ and intellectual property: tools for development}

Aura Esther Troconis Troconis

Universidad Simón Bolivar, Venezuela

Autor para correspondencia: atroconis@usb.ve

Fecha de recepción: 24 de Abril de 2017 - Fecha de aceptación: 10 de Julio de 2017

Resumen: El creciente reconocimiento que ha alcanzado el conocimiento como un factor influyente y un indicador relevante al momento de generar y evaluar las estrategias vinculadas al desarrollo económico, social y cultural de las naciones, y con ello, el rol que desempeñan las políticas públicas como los mecanismos para lograr su enlace con la sociedad, fundamentan el propósito de esta investigación. En este sentido las políticas públicas vinculadas al conocimiento son aquellas afines a los conceptos de ciencia, tecnología e innovación $(\mathrm{CT}+\mathrm{i})$, factores que definen los procesos de creación, transformación y novedad de éste.

En el mundo de los intangibles, de las ideas, del conocimiento, no se genera automáticamente el crecimiento económico y desarrollo; este valor se genera cuando existe en los países un esqueleto estructural conformado por un sistema de políticas públicas que institucionaliza, legaliza, concientiza y protege las acciones a seguir en el marco de la ciencia, la tecnología y la innovación, con lo cual, es posible dar el paso de las ideas a la transformación en activos económicos o bienes tangibles. Este análisis busca establecer la importancia y vinculación que existe entre las políticas públicas de ciencia, tecnología e innovación y las políticas en propiedad intelectual como un instrumento clave para el progreso de los países y con ello, una mejor calidad de vida para los ciudadanos.

Palabras claves: políticas públicas; CT+i; propiedad intelectual; desarrollo

\begin{abstract}
The growing recognition that knowledge has reached as an influential factor and a relevant indicator when generating and evaluating the strategies linked to the economic, social and cultural development of nations, and with it, the role played by public policies such as mechanisms To achieve their link with society, underpin the purpose of this research. In this sense the public policies linked to knowledge are those related to the concepts of science, technology and innovation (CT $+\mathrm{i})$, factors that define the processes of creation, transformation and novelty of the latter. In the world of intangibles, ideas and knowledge, economic growth and development are not automatically generated; This value is generated when there exists in the countries a structural skeleton made up of a system of public policies that institutionalizes, legalizes, conscientizes and protects the actions to be taken in the framework of science, technology and innovation, which is possible Give the passage of ideas to the transformation into economic assets or tangible goods. This analysis seeks to establish the importance and linkage between public policies on science, technology and innovation and intellectual property policies as a key instrument for the progress of countries and with that, a better quality of life for citizens.
\end{abstract}

Key words: public policies; CT + I; intellectual property; development 


\section{Introducción}

Las políticas públicas van mucho más allá de la expresión e interpretación de los intereses de los actores y de las instituciones. En ella intervienen la teoría de las ideas. En ese sentido, Sanz y Borras (2000), destacan como a partir de las ideas se generan grandes aportes a los enfoques institucionales de la economía política y las relaciones internacionales, contribuyendo de manera significativa a aclarar las cuestiones conceptuales relacionadas con los efectos de las ideas sobre la formulación de políticas, la explicación de la visión que se tiene del mundo, la creación de un modelo causal, o un cambio de paradigma como el que ocurrió con la teoría económica de Keynes en su momento.

Campbell (citado en Sanz y Borras, 2000: 3), hizo un intento por definir una tipología de las ideas en base a dos dimensiones que denominó: "la normativa que (consiste en los valores y las actitudes) y la cognitiva (que da estructura a las ideas transformando el conocimiento en argumentos), y en segundo lugar, la distinción analítica entre el 'primer plano' (el normativo) y la deliberación política", con lo que podría decirse inicialmente, que las políticas públicas en CT+i expresan la relación entre las ideas, los intereses de los actores y las formas en que los problemas son estructurados.

De esta manera, se genera la vinculación a las políticas públicas de un concepto clave para la evolución de las sociedades y sus modelos de desarrollo, este es, la innovación y su consideración como el marco para aglutinar la acción no sólo de los entes públicos, sino también, de los institutos de I+D+i, de las universidades públicas y privadas, de las empresas públicas, las empresas privadas, laboratorios de I+D, innovadores, parques tecnológicos, en fin, de todos aquellos actores que ejercen las funciones de ciencia, tecnología e innovación en cada país alrededor del mundo.

Se incorpora una nueva tarea para los Estados y la sociedad en general, basada en la creación de estructuras políticas e instrumentos que soporten las actividades CT+i más allá de un nivel meramente cognitivo, en otras palabras, se deben definir las políticas públicas para cada uno de estos sectores.

En relación a las políticas de ciencia y tecnología, Albornoz (1997:1), las define como “el despliegue de capacidades de innovación de la sociedad mediante la vinculación entre diversos actores (fundamentalmente el gobierno, los individuos e instituciones científicas o académicas y las empresas)". Capacidades supeditadas a los procesos a partir de los cuales, se generan las trayectorias para alcanzar los objetivos del sistema de innovación, que tiene su origen en las ciencias básicas, hasta lograr su implementación en la sociedad a través de la tecnología.

Por otro lado, Ramón y Collado (2012: 147), añaden al concepto de las políticas de ciencia y tecnología, la idea de cómo éstas indican al..."gobierno aquellas direcciones que tienen como finalidad resolver los problemas importantes de un país...". Este enfoque guarda relación con el valor que la ciencia y la tecnología infringe a los procesos de desarrollo, direccionados sobre todo a la disminución de las desigualdades sociales y a la tarea del gobierno de generar mejores condiciones de vida para la población, por lo que, la ciencia y la tecnología se transforman en herramientas claves para ello. 
Otra visión del concepto de políticas de ciencia y tecnología, es la presentada por Salazar (2010: 57), al referirse a éstas de manera separada, definiendo a la Política Científica, como aquella que está ... "destinada a estimular la generación, transmisión y difusión del conocimiento científico dentro y fuera del mundo de la academia"... Mientras que la... "Política Tecnológica, se formula para la generación y adquisición de tecnologías y destrezas con fines de estimular la industria, sean incentivos para la exportación (draw back), reinversión en actividades de I \&D (exoneración de impuestos para el desarrollo de actividades privadas de I \&D)”.

En la primera, la política se enmarca como la referencia del origen del conocimiento hasta su multiplicación por medios concebidos en el espectro de la academia, como lo son, la docencia, la investigación y la extensión, mientras que, en el segundo plano, se habla del desarrollo de las herramientas que serán utilizadas por un tercero, en este caso, la industria para lograr multiplicar este conocimiento en factores de producción.

Todas las posiciones señaladas, recalcan el valor de los conceptos de ciencia y tecnología y de las políticas referentes a éstas, como elementos preponderantes en las decisiones que encuadran la direccionalidad y utilidad del conocimiento en las sociedades, y a su vez, como el esqueleto que le da estructura a un concepto intangible cada vez más influyente en la actuación de los gobiernos, así como también, en las relaciones comerciales e internacionales.

Hacia finales del siglo XX e inicios del siglo XXI se comienza a hablar de una nueva forma de generar el conocimiento científico, con una amplia participación de actores, se incluye a las redes de conocimiento, que se involucran en los procesos de toma de decisiones en materia científica y tecnológica de la mano con el gobierno, con la característica de que éstos participarán en la política pública de principio a fin.

Por tales razones, hoy en día al proferir sobre políticas públicas de ciencia y tecnología, estas van de la mano de las políticas públicas de innovación, las cuales pueden definirse como:

La acción-respuesta de los actores sociales- Estado, Universidad, (Academia), Industria, Sociedad Civil (ciudadanos)- en la búsqueda de la mejor estrategia para la satisfacción de sus necesidades endógenas o exógenas, derivadas de su interactuar cotidiano con la sociedad y en la sociedad, e insertarse en su comunidad internacional (la aldea global) (Salazar, 2010: 57).

Y con ello, debe hacerse énfasis en los procesos de aprendizaje, la difusión e incremento de la complejidad del conocimiento, del mismo modo, que la relevancia que juega el individuo como el activo que da vida a este proceso, todos como elementos claves de esta política.

En la medida que estas redes se construyan y fortalezcan en los países, se estará en capacidad de multiplicar y asentar esas condiciones favorables para generar ventajas innovativas nacionales, que no es más que, crear las bases apropiadas para el desarrollo de los procesos de innovación, y asumirlas "como un problema estratégico de Estado, el cual se compromete a estimular promover y proteger la creatividad y la inventiva nacional, y crear las condiciones para crear una cultura sustentada en la innovación" (Salazar, 2010: 63). 
Las consecuencias de este cambio de paradigma en la formulación de las políticas públicas para la innovación, es indicativo de que los tipos de medidas consideradas por los responsables políticos deben ampliarse más allá del tradicional apoyo económico e institucional a la I + D, y por el contrario, la política pública en innovación debe adoptar un enfoque de carácter sistémico y sostenible en el tiempo.

Lo cual es posible, según Silva (2005: 99), a partir de la correlación de tres elementos claves que surgen alrededor de este proceso, a saber: "la solidez institucional, la continuidad y la coordinación de las políticas en diversas áreas estratégicas”. Incluyendo, la consideración de las particularidades que forman parte de cada sistema de innovación, al tipo de sociedad, al enfoque, la cultura de innovación implantada, las características de los sectores y actores involucrados y la estructura y coyunturas país, entre otros elementos que finalmente contribuyen a la generación y selección de los mecanismos y estrategias más apropiadas para ser llevados a cabo.

Es de resaltar, que todos los aspectos descritos, así como, las relaciones presentes entre los mismos, forman parte de lo que se denomina Sistemas Nacionales de Innovación (SIN), concepto acuñado hacia finales del siglo XX, que se incorpora a las discusiones sobre políticas públicas en el sector científico - tecnológico para dar forma y sustentación al escenario de las relaciones propias entre y de los actores, funciones, actividades y decisiones alrededor de la ciencia, la tecnología y la innovación.

Además, el enfoque de sistema de innovación se ha ido acercando cada vez más a la economía, dejando en claro, que su contribución al desarrollo económico de los países es mucho mayor que simplemente la creación y reforzamiento de las instituciones encargadas de las políticas públicas.

Esto se observa en el concepto sobre sistemas de innovación generado por Lundvall (1992), al explicarlo como la constitución de un:

...conjunto de elementos y sus interrelaciones, que intervienen en la producción, difusión y uso de conocimiento nuevo y económicamente útil y es nacional puesto que comprende los elementos e interrelaciones localizados dentro de las fronteras o arraigadas en la nación estado. (Lundvall, citado en Sánchez y Estrada, 2001: 10).

De acuerdo a lo citado, los sistemas de innovación son una herramienta que beneficia a los procesos económicos, y permiten identificar los aspectos que deben destinarse en favor del desarrollo y valoración de la innovación, como un factor altamente diferenciador entre las naciones del mundo.

Aunado a todo lo anteriormente mencionado, es importante resaltar que inmerso en la evolución y aplicación de estos conceptos y el desarrollo de políticas públicas, se encuentra vinculada la protección de los derechos sobre los bienes inmateriales a través de la propiedad intelectual y los diferentes instrumentos administrativos y/o legales, que forman parte de este proceso, convirtiéndose para los gobiernos alrededor del mundo, en un elemento clave, y junto a ello, la generación de políticas públicas como soporte de los sistemas de propiedad intelectual, 
complemento lógico de cualquier estrategia de desarrollo industrial y tecnológico en el plano internacional.

Dichos sistemas deben contribuir en la construcción y mantenimiento de ambientes favorables para la generación de conocimiento, propiciando la innovación, que promuevan y protejan las inversiones, y principalmente, que estimulen la cooperación entre los diferentes actores de los sistemas nacionales e internacionales de $\mathrm{CT}+\mathrm{i}$.

Estos argumentos dan paso al análisis relativo a la vinculación de las políticas públicas en $\mathrm{CT}+\mathrm{i}$ y las políticas públicas en propiedad intelectual, temas que soportan el objetivo que se persigue en esta investigación, como factores altamente influyentes en el desarrollo económico, comercial, social, y cultural de las naciones inmersas en la sociedad del conocimiento.

Parte de las políticas públicas en CT+i son las políticas en propiedad intelectual, ambas están íntimamente vinculadas, debido a que en las primeras se hace énfasis en la creación, desarrollo e innovación sobre el conocimiento, y en las segundas, se generan las decisiones, los mecanismos, instrumentos, servicios, infraestructura, para facilitar la protección y la incorporación de valor a la producción nacional de bienes y servicios a través de éste, en aras de contribuir con el desarrollo económico y productivo, y que ello redunde no sólo en un mejor nivel de vida para la población, sino también, en la generación de estrategias y ventajas competitivas país frente a otras naciones del mundo.

La propiedad intelectual y las políticas públicas que se generan alrededor y a partir de ésta, deben integrarse como un componente activo de los sistemas de innovación nacional, lo cual es posible mediante la atención a una serie de iniciativas que se emprenden y están condicionadas a las características de cada sociedad, y al valor que hayan alcanzado los conceptos de ciencia, tecnología e innovación en éstas, pero también, dependen de procesos coyunturales que puedan profundizar positivamente este valor o transformarlo en un obstáculo.

En ese sentido, algunas de estas iniciativas corresponden a las que enuncia Díaz (2006: 134-135), como:

a) "Eliminar trabas innecesarias para proteger resultados de $\mathrm{I}+\mathrm{D}+\mathrm{i}$.

b) Formar en gestión de PI a investigadores.

c) Promover entidades de transferencia tecnológica.

d) Establecer en general criterios rigurosos de patentabilidad.

e) Desarrollar sistemas estadísticos y sistemas de indicadores, entre otras".

Las políticas públicas en propiedad intelectual están destinadas entre sus múltiples objetivos a estimular la innovación y la creación, ofreciendo la perspectiva de una recompensa no sólo monetaria que permitirá a los titulares recuperar las inversiones en investigación y desarrollo (I + D) realizadas, sino también, la protección de un derecho moral, de reconocimiento sobre quienes ejercen ese derecho, lo cual se ha ido incrementado, en la medida que las economías del mundo se desarrollan y adquieren activos de conocimiento valiosos para lograr este objetivo. 
En consideración a esto, las naciones se han volcado en gran medida a la generación de instrumentos, por medio de los cuales, los derechos de propiedad intelectual encuentren una mayor organización, sentando bases sólidas en la formulación de sistemas políticas públicas que formen parte de la negociación e intercambio de los activos intangibles, sustentados en primer lugar, a nivel nacional para a posteriori, poder ejercer estos derechos internacionalmente.

En otras palabras, tanto las políticas públicas en $\mathrm{CT}+\mathrm{i}$ como las políticas públicas en propiedad intelectual deben abordarse en forma sistémica, lo cual implica constituir un balance entre los incentivos a los innovadores con los intereses de la sociedad, alcanzando maximizar la difusión social del conocimiento y el desarrollo económico.

Estas políticas han pasado a ocupar un rol de mayor alcance dentro del hemisferio de la ciencia, la tecnología y la innovación, producto de la vorágine de cambios que se han generado en las economías y las formas de establecer las relaciones internacionales en el mundo, haciendo que las políticas en la materia pasen de ser un actor secundario utilizado por otros sectores para tratar asuntos legales vinculados a la protección del conocimiento, a transformarse en protagonista de primera línea, con respecto a la definición, interpretación e inclusive negociación del conocimiento como un valor estratégico.

Los derechos de la propiedad intelectual expresados entre otras herramientas a través de las políticas públicas, se convierten en el soporte de sistemas culturales para la innovación que caracterizan a las naciones competitivas, como aquellas que descansan sobre los cimientos de actividades de investigación y desarrollo científico - tecnológicas, que deben conducir a la adopción de los mejores mecanismos para la protección de los resultados tangibles provenientes de esas invenciones, garantizando el posicionamiento de los mismos en las fronteras internas y en un mediano y largo plazo hacia las fronteras y mercados internacionales.

En el nuevo paradigma de la innovación, las políticas públicas de propiedad intelectual se han transformado en un vehículo que va desde la transferencia del conocimiento a la protección del mismo, por lo tanto, invariablemente los cambios que se produzcan en los sistemas de innovación también afectaran lo que se exige de los sistemas de propiedad intelectual.

Algunos de los factores que se han identificado y son descritos por la Organización Mundial de Propiedad Intelectual (1992) para explicar este proceso, corresponden a un conjunto de condiciones en la que se genera una manifiesta interrelación entre las políticas de ciencia, tecnología e innovación con las políticas de propiedad intelectual, a saber:

a) Aumento de la inversión en I+D y cambios en la propensión a patentar, es decir, en generar constantemente invenciones susceptibles de patentamiento en sus diversas formas.

b) Crecimiento del número de solicitudes de patentes posteriores a la patente primaria, con lo cual se da continuidad y mayor vida útil de explotación en el mercado a la invención generada inicialmente, debido a que se generan invenciones menores derivadas de esta.

c) Ampliación de posibilidades tecnológicas, que aluden a tecnologías disruptivas o tecnologías de punta que se han insertado como nuevas áreas de conocimiento. 
d) Cambios jurídicos e institucionales, que se relacionan con la creación de nuevas instituciones, políticas y legislaciones nacionales e internacionales, que promueven los derechos de propiedad intelectual como parte del desarrollo económico y social.

e) Patentamiento estratégico, o lo que se denomina estrategias de patentamiento defensivas para proteger los mercados nacionales e internacionales de la penetración o aparición de competidores que inunden el mercado de nuevos productos, tecnologías, procedimientos, etc., con lo cual se busca obstaculizar al competidor.

A lo cual se suman otros instrumentos como las políticas, normativas y legislaciones relativas a: los ingresos y los pagos por concepto de regalías y derechos de licencia por el uso de activos intangibles; el comercio de servicios relacionados con la tecnología; los ingresos y los pagos por la prestación de servicios de I+D; exportaciones de tecnología y de servicios de I+D; procesos de transferencia de tecnología; asociaciones estratégicas; negociaciones de regalías o royalties y sub contratación de actividades de I+D.

Otras estrategias que algunos Estados y el sector privado han desarrollado en diversos países, han orientado las políticas en la materia a la búsqueda de talento investigador ofreciéndoles mejores condiciones para desarrollar estas actividades, dejando desprovistas a otras naciones de masa crítica que sustente la actividad de $\mathrm{I}+\mathrm{D}+\mathrm{i}$ en sus países. Estas acciones pueden estar relacionadas con la necesidad de acelerar el fortalecimiento de capacidades del talento humano; la ampliación del espectro conformado por los trabajadores del conocimiento, con el objetivo de mejorar la capacidad tecnológica para absorber y adaptar las tecnologías importadas a través de la inversión extranjera directa con lo cual se produzca una mayor liberalización del mercado; pero, para que esto tenga el efecto deseado es necesario proporcionar entre otros factores un marco institucional y políticas de negocios que sustenten estos procesos.

Cuando se habla de un marco institucional adecuado éste va mucho más allá que simplemente pensar en el financiamiento. Se requiere de políticas públicas, un clima y una cultura de apoyo de la I+D+i; donde exista un equilibrio entre el aprovechamiento del mercado y la proyección y multiplicación de las buenas prácticas, para identificar los mejores productos y servicios a introducir en el mercado, y que éstas acciones formen parte de la manera más eficientemente posible, de los programas implementados por los gobiernos.

Cuando el sistema de protección de los derechos de propiedad intelectual funciona como se pretende y cumple con los objetivos políticos establecidos para ello, se genera un valor incalculable de dicho mecanismo como un factor para estimular y apoyar el clima país idóneo, que permitirá que los frutos de la innovación retribuyan a las sociedades múltiples beneficios.

Entre los indicadores más fiables y resaltantes para expresar el estatus de la innovación de un país o región, está precisamente la actividad de patentes. Las patentes son una medida clave del éxito de una verdadera cultura de $\mathrm{I}+\mathrm{D}+\mathrm{i}$ debido a que éstos reflejan en gran parte la capacidad inventiva de las sociedades, son un importante insumo que contribuye con la difusión del conocimiento, y son buenos indicadores comparativos del nivel de internacionalización, regionalización y la nacionalización de actividades de innovación de los países. 
Todo lo mencionado anteriormente corresponde al estado ideal en la combinación entre crecimiento económico, la innovación y la propiedad intelectual, en síntesis, la vinculación entre las políticas de $\mathrm{CT}+\mathrm{i}$ y propiedad intelectual. Encontrar y mantener este equilibrio ha sido la fórmula que pocos países disfrutan, y que otros siguen intentando apalancar en sus proyectos de desarrollo.

Por lo antes expuesto, el éxito y el valor agregado deberán estar fundamentados en la combinación de la gestión empresarial y la gestión del sector público, la acción de los diversos actores que componen los Sistemas Nacionales de Ciencia, Tecnología e Innovación (SNCTI), con el sistema de propiedad intelectual. La figura 1 a continuación resume esquemáticamente estos aspectos:

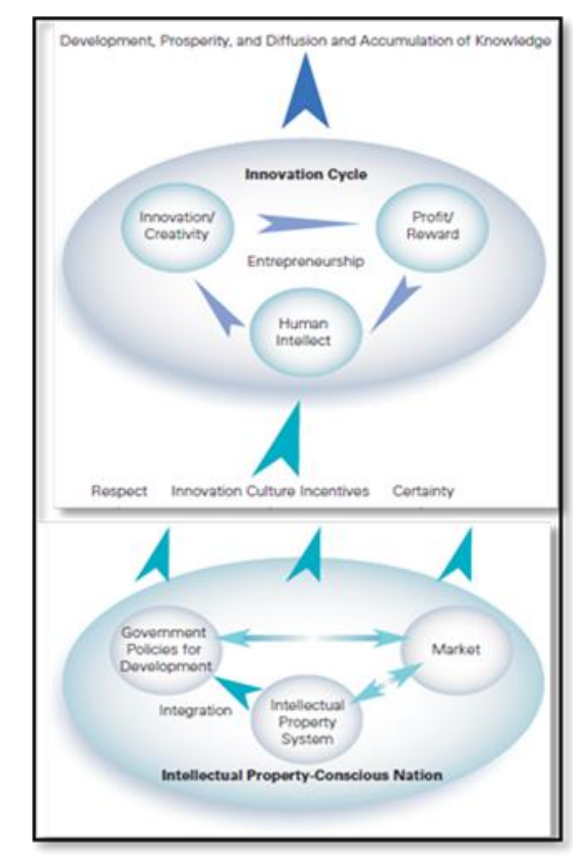

Figura 1. El Ciclo de la Innovación en una Nación consciente de la Propiedad Intelectual.

Idris y Arai. (2005). The Intellectual Property-Conscious Nation: Mapping The Path From Developing To Developed. Ginebra: OMPI.

Este esquema representa el concepto subyacente que define a una cultura de innovación con bases firmes, en la que el uso, la inclusión y la integración del sistema de propiedad intelectual en las políticas gubernamentales y las estrategias a partir de las cuales se implementan esas políticas, ayudarán a transformar a la innovación en bienes transables, económicos que puedan generar ganancias financieras.

Estos activos como en un ciclo pueden ser utilizados y reutilizados con fines comerciales, para fomentar la innovación y la creatividad, contribuyendo con la creación de riqueza, y aumentar el acervo de conocimientos y las reservas de la nación favoreciendo la creación y afianzamiento de una dinámica autosostenible en materia de I+D+i, construyendo el puente entre la innovación y el mercado. 
Pero de la misma manera, como existe una relación intrínseca entre la protección de la propiedad industrial y el desarrollo económico desde la visión del mercado, también existe un beneficio producto de la vinculación de las políticas públicas en CT+i y propiedad intelectual basadas en el conjunto de relaciones ganar-ganar que se establecen a partir de estos eslabones (conocimiento+innovación+propiedad intelectual), basadas en la confianza y el cuidadoso equilibrio de la creación de un nuevo conocimiento y la influencia que este proceso puede ejercer sobre el desarrollo de asociaciones y alianzas estratégicas intra y extra fronteras, que le otorgan a la protección de los derechos de la propiedad intelectual un papel preponderante en el juego de las relaciones internacionales y en la concientización del conocimiento como un valor país.

Este proceso tiene que ver con la generación de activos internos representados principalmente por el capital intelectual y relacional, así como, las motivaciones e incentivos, a la idea de aprender y aprehenderse de la ciencia, tecnología e innovación y su protección como procesos naturales en los individuos, en recompensas a su contribución intelectual y en la multiplicación de este valor como un referente país, porque se está protegiendo la inventiva humana independientemente de que éstas puedan o no llegar al mercado comercial.

\section{Conclusiones}

Puede decirse entonces, que la esencia de los derechos de propiedad intelectual, y su función en el crecimiento económico y desarrollo de los países radica en hacer valer los derechos del conocimiento, todo en una frase, empoderarse del conocimiento como uno de los motores propulsores del desarrollo, este reto exige un fuerte compromiso al más alto nivel de gobierno, que debe ser seguido por políticas, estrategias, planes y acciones claramente delineados, basados en la eficiencia, la coordinación y la cooperación entre los actores que integran el sistema de innovación, con el fin de encontrar el mejor equilibrio entre los diferentes intereses, conforme a las necesidades y el nivel de desarrollo de cada país.

En este orden de ideas, Idris y Aray (2005: 98), señalan que:

...asegurar que las políticas de propiedad intelectual se integren en la estrategia nacional de desarrollo, y lograr un equilibrio adecuado entre los objetivos de esa política pública y los incentivos y la infraestructura que la componen, se convierten en el combustible para la innovación y la creatividad.

Ese proceso de integración requiere de constantes ajustes dadas las cambiantes necesidades de las sociedades, ajustes en cuanto a sus políticas públicas y los recursos e instrumentos para hacerlas posible. Como gobiernos proactivos en este tipo de procesos, deben generarse programas de sensibilización sobre la propiedad intelectual como una herramienta clave en pro del desarrollo, sistemas de propiedad intelectual que brinden administrativa, legal y financieramente apoyo a los actores del sistema de innovación, así como otras iniciativas que proyecten a la propiedad intelectual como un instrumento de protección y expansión de la ciencia, la tecnología y la innovación.

Estas decisiones implican alimentar al sistema de innovación, generando a partir de las políticas públicas en propiedad intelectual un músculo sustentado sobre talantes como: 
a) Una cultura que favorezca la innovación y la creatividad y a su vez, sea capaz de luchar contra dos grandes flagelos de la propiedad intelectual, como lo son la falsificación y la piratería.

b) Generar capacidades nacionales y desarrollar talento humano para la gestión de activos de propiedad intelectual, reforzando la participación de actores como las universidades, las instituciones públicas y privadas de investigación y desarrollo, laboratorios, emprendedores, cultores, parques tecnológicos, etc.

c) Y acompañar a los conocimientos provenientes de la ciencia, la tecnología y la innovación del conocimiento tradicional y el patrimonio cultural de los países.

Sin embargo, estos procesos a lo largo de los años y específicamente luego del surgimiento de la Organización Mundial de la Propiedad Intelectual (OMPI) en 1967, alcanzaron niveles que traspasaron las fronteras nacionales, generándose la internacionalización de los derechos de la propiedad intelectual y con éstos, la creación de instituciones, legislaciones, programas e iniciativas, enlazadas con la política exterior de los países mediante acuerdos, tratados, la adhesión a entes y alianzas con el objeto de universalizar éstos derechos, y a través de ello, construir nuevos puentes para la innovación. En consecuencia se genera una visión global de los derechos de propiedad intelectual, y de los actores que la hacen posible.

Los distintos instrumentos que surgieron con la idea de universalizar los derechos de propiedad intelectual corresponden a una serie de leyes y tratados en la materia; clasificaciones y normas internacionales, versadas sobre la protección, y el registro. Entre los más importantes se encuentran los siguientes:

a) Tratado de Beijing sobre interpretaciones y Ejecuciones Audiovisuales.

b) Convenio de Berna.

c) Convenio de Bruselas.

d) Arreglo de Madrid (Indicaciones de procedencia y marcas).

e) Tratado de Marrakech para las personas con discapacidad visual.

f) Convenio de París.

g) Convenio de Fonogramas.

h) Convenio de Roma.

i) Tratado de Singapur sobre el Derecho de Marcas.

j) Tratado de Washington.

k) Tratado de Budapest.

1) Arreglo de La Haya.

m) Arreglo de Lisboa.

n) Arreglo de Niza.

o) Arreglo de Locarno.

p) Arreglo de Estrasburgo.

Estos instrumentos de tipo orientador, buscan homologar el trato de los derechos de propiedad intelectual internacionalmente, pero, ello no implica que los países sumados a los mismos pierdan o revoquen toda capacidad de ejercer su soberanía en la materia. De alguna forma, las naciones que se suman a estos instrumentos buscan vincular su espacio nacional, sus 
políticas públicas en $\mathrm{CT}+\mathrm{i}$ y en materia de propiedad intelectual con las organizaciones y entes internacionales y por medio de ello, participar, ganar y fortalecer espacios extra muros conectados y resguardados a través de las dimensiones que comprenden una de las herramientas sobre las cuales se sustentan las relaciones internacionales de un país, su política exterior.

Dichas legislaciones han evolucionado en respuesta a los avances de la ciencia, la tecnología y la innovación en comunión con los derechos de propiedad intelectual, con el objetivo de apoyar el desarrollo de los mercados nacionales en aras de inspirar la competitividad que responda a los requerimientos que el mercado internacional ha impuesto en la materia, reconociendo el nivel de protección más apropiado a las innovaciones tecnológicas.

El conocimiento se ha vuelto un mecanismo más de vinculación entre las naciones, desde la perspectiva positiva, como una forma de ganar - ganar en la que el beneficio es compartido por aquellos que lo generan; pero también, puede ejercerse como una ventaja orientada a la dominación, intervención, manejo o apropiación del conocimiento en perjuicio del otro, ejerciendo el poder para imponer y no para negociar.

El conocimiento se convierte entonces, en un factor determinante entre las características que distinguen a ese modelo de sociedades, con la prerrogativa de que el "acceso universal, masivo, intensivo y permanente a los conocimientos existentes y los que se van generando" (Ávalos, 2005: 23), debe ser una constante, acompañado de políticas públicas, herramientas e instrumentos administrativos y legales que lo fortalezcan mediante la actuación conjunta de todos los actores llamados a ellos, que pasan desde el ciudadano común como ente participante en el proceso de formulación de la política y como receptor de la misma, pero también de instituciones públicas, privadas, universidades, institutos de investigación y desarrollo, emprendedores, innovadores populares, parques tecnológicos, laboratorios de I+D, etc.

Lo anteriormente descrito muestra una vez más, que el alcance y la vinculación entre las políticas públicas en $\mathrm{CT}+\mathrm{i}$ y las políticas públicas en propiedad intelectual es cada vez mayor y se superpone a otros ámbitos del quehacer de los países, inclusive como un mecanismo de negociación y posicionamiento de las naciones desde la perspectiva comercial, la generación de alianzas estratégicas a nivel de negocios y formación, transferencia de tecnología, posicionamiento de marcas en diversidad de mercados, en el marco de la generación de lo que se denomina las fronteras invisibles, establecidas por el conocimiento y su protección como una fuente de ventajas competitivas sostenibles.

\section{Bibliografía}

Albornoz, M. (1997). La política científica y tecnológica en América Latina frente al desafío del pensamiento único. Revista Redes, vol. 4 - 10, Argentina, Universidad Nacional de Quilmes, 95-115.

Ávalos, I. (2005). "La investigación universitaria en tiempos de la sociedad del conocimiento". Revista Venezolana de Economía y Ciencias Sociales, vol.11 no.1. Obtenido de: http://www.scielo.org.ve/ 
Díaz, A. (2006). TLC y Propiedad Intelectual: Desafíos De Política Pública en 9 países de América Latina y el Caribe. Brasil: CEPAL.

Idris, K., \& Arai, H. (2005). The Intellectual Property-Conscious Nation: Mapping the Path from Developing To Developed. Ginebra: OMPI.

Lundvall, B. (1992). National System of Innovation: Towards a Theory of Innovation and Interactive Learning. Londres: Pinter.

Organización Mundial de la Propiedad Intelectual - OMPI. (1992).Qué es la Propiedad Intelectual? Ginebra: OMPI.

Ramón, F., \& Collado, R. (2012). Hacia el establecimiento de políticas de ciencia e innovación en América Latina. Papel de los organismos latinoamericanos de ciencia e innovación. Revista Nuevo Mundo, Año IV N 8, Caracas, 143 - 173.

Salazar, L. (2010). El Circuito Jurídico Económico de la Propiedad Intelectual. Caracas: CENDES.

Sánchez, M.P., \& Estrada, S. (2001). Sistema Nacional de Innovación. (Biblioteca de la Universidad Autónoma de Madrid). Madrid: Autor.

Sanz, L., \& Borras, S. (2000). Explaining changes and continuity in EU technology policy: The politics of ideas. Papel de trabajo del Institute for Advanced Social Studies, Madrid (Spain) and Roskilde University. Dinamarca: CSIC Unidad de Políticas Comparadas and Roskilde University. 